\title{
Surface nano-alloying treatment of low carbon steel
}

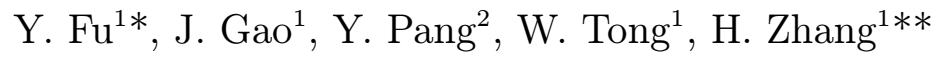 \\ ${ }^{1}$ Key Laboratory of Electromagnetic Processing of Materials, Ministry of Education, Northeastern University, Shenyang, \\ Liaoning 110004, P. R. China \\ ${ }^{2}$ Jiangsu Yancheng Environmental Protection Tech City Rapid Solidification and Additive Manufacturing Engineering \\ Technology Center, Yancheng 224001, P. R. China
}

Received 19 November 2019, received in revised form 2 April 2020, accepted 22 April 2020

\begin{abstract}
A nano-structured alloying layer was fabricated by the addition of 304 stainless steel powder to the surface of a low carbon steel (LCS) plate by surface mechanical attrition treatment (SMAT). The coverage of the stainless steel layer was $93 \%$ after SMAT for $60 \mathrm{~min}$. The thickness of the surface layer was approximately $35 \mu \mathrm{m}$. The average grain size in the top layer was in the range of $55-65 \mathrm{~nm}$ with random crystallographic orientation, and the grain size increased with increasing depth from the treated surface. Both the surface hardness and wear resistance of the sample that underwent a surface nano-alloying treatment (SNAT) increased compared with those of the LCS sample. Additionally, the wear resistance increased for the nanocrystalline (nc) surface layer. Nanoindentation measurements indicated that micro-hardness in the nc surface layer was approximately $645 \mathrm{HV}$, which was three times as high as that of the LCS matrix.
\end{abstract}

K e y words: surface nano-alloying treatment (SNAT), surface mechanical attrition treatment (SMAT), microstructure, 304 stainless steel layer, micro-hardness

\section{Introduction}

Low carbon steel has been widely used in various industries due to its advantages, such as good plasticity, good processing performance, and low-cost production; however, it also possesses low hardness, low corrosion resistance and poor surface wear resistance, which restricts its further application. In most cases, failures of low carbon steels initiate at the surface and are sensitive to the microstructure and properties of the surface. It is well known that nc materials have excellent physical and mechanical properties, such as a large volume fraction of grain boundaries, high hardness, and strong wear resistance, which have attracted considerable interest [1]. It is reasonable to modify the surface of a material to enhance its performance properties by the generation of an nc surface layer, in which the chemical composition remains unchanged relative to the matrix. This is the so-called self-nanocrystallization approach. Such surface nanocrystallization can be easily realized by means of specific conventional surface treatment techniques, such as ultrasonic shot peening and surface mechanical attrition treatments (SMATs) [2-4]. Recent studies have shown that nc layers can be produced on various metallic materials by means of a SMAT $[5,6]$. This is an effective surface modification technique to improve surface properties, including hardness and wear resistance [7-11]. After a SMAT, atomic diffusion is significantly enhanced due to a large number of grain boundaries and dislocation substructures, which can act as fast atomic diffusion pathways [12]. The original grains are gradually refined to the nanometre size regime, and different microstructures can be obtained within the deformed surface layer from the treated surface to the strain-free matrix [13-16]. Therefore, the formation of an nc layer on the surface of LCSs is an effective method to enhance the atomic diffusion kinetics. For example, Sun et al. fabricated a micro-alloy nitrided layer on an LCS by nitriding combined with a surface nano-alloying pretreatment [17]. P. Magudapathy et al. studied the alloying

\footnotetext{
*Corresponding author: e-mail address: fyzh1224@163.com

**Corresponding author: e-mail address: hzhang@epm.neu.edu.cn
} 
of metal nanoparticles by ion-beam-induced sputtering [18]. Intermetallic compounds of the $\mathrm{Ti}-\mathrm{Al}$ system have been considered extensively for high-temperature structural applications because they offer a combination of good oxidation resistance and useful mechanical properties at temperatures higher than those possible with conventional titanium alloys. The optimum surface properties should not conflict with other requirements, such as low material cost, ease of fabrication and high strength. S. Romankov et al. introduced the mechanical alloying method for the manufacturing of Ti-Al coatings [19].

In this paper, an LCS was used to study surface nano-alloying treatment. A nano-alloy coating of 304 stainless steel was synthesized on the LCS surface. The alloy layer was found to have high hardness and good wear resistance. The microstructure and hardness variation at different depths of the alloy layer of the treated sample were characterized by optical microscopy (OM), X-ray diffraction (XRD), scanning electron microscopy (SEM), energy dispersive spectrometry (EDS), transmission electron microscopy (TEM), and Vickers hardness testing. The friction and wear properties were investigated and compared with those of the coarse-grained sample. The effects of grain refinement on the friction and wear properties of the surface layer are discussed.

\section{Experimental methods}

The material used in the present study was a commercial LCS bar with a diameter of $50 \mathrm{~mm}$ and 304 stainless steel powder. The chemical composition of the LCS was (in wt.\%): C 0.20, Si 0.20, Mn 0.50, $\mathrm{Cr} 0.20, \mathrm{Ni} 0.10, \mathrm{~S} 0.02, \mathrm{P} 0.02, \mathrm{Cu} 0.20$, and balance Fe. The particle size of the 304 stainless steel powder was 200 mesh, and the chemical composition was (in wt.\%): C 0.05, Si 0.80, Mn 1.50, Cr 17.00, Ni $10.20, \mathrm{~S} 0.02, \mathrm{P} 0.02, \mathrm{Cu} 0.02$, and balance Fe. The LCS sample was first annealed at $600^{\circ} \mathrm{C}$ for $1 \mathrm{~h}$ in vacuum to release the residual stress and relieve the effects of mechanical deformation to obtain a homogeneous and coarse grain structure. Additionally, the LCS bars were cut into sheets with dimensions of $\varnothing$ $49 \mathrm{~mm}$ and thickness of $3 \mathrm{~mm}$ by wire-cut electrical discharge machining (WEDM). Before the SMAT, the LCS plates were polished with 60 to 3000 grit abrasive papers to remove the WEDM marks. Finally, the LCS sheets were ultrasonically cleaned in acetone for $10 \mathrm{~min}$. The surface nano-alloying treated sample was produced in a vacuum chamber. The SNAT process was performed by using a SPEX/8000M mill $\left(\mathrm{SPEX}^{\circledR}\right.$ SamplePre Metuchen, NJ, USA) with a rotation speed of $875 \mathrm{rad} \mathrm{min}^{-1}$. The LCS sheet was fixed at the top of the cylinder-shaped vacuum chamber. Several GCr15 steel balls that were $8 \mathrm{~mm}$ in diameter and approximately $1 \mathrm{~g}$ of the 304 stainless steel powders were placed at the bottom of the vacuum chamber. The SNAT process was performed in a protective atmosphere of high-purity argon $(99.999 \%)$ to prevent the oxidation caused by the temperature increase during ball grinding. The vacuum chamber was vibrated by a high-speed generator with a frequency of $50 \mathrm{~Hz}$. In the chamber, the balls resonated, and one side of the sample surface was peened repeatedly and multidirectionally. The balls generated mechanical attrition on the surface of the LCS sheet, causing severe plastic deformation (SPD) in the surface layer and welding of the 304 stainless steel powder to the surface of the LCS sample. In this work, the SNAT time was $60 \mathrm{~min}$.

The microstructure of the surface layer in the treated samples was characterized by a Leica DMI 5000M metallographic microscope. The cross-sectional morphology observation and corresponding energy dispersive analysis of the surface nano-alloying treated samples were conducted by using field-emission scanning electron microscopy (SEM; Zeiss Ultra 55 PLUS). The grain size, micro-strain, and phase information of the nc layer were obtained by using X-ray diffraction (XRD; X'Pert Pro PW3040/60, PANalytical, Lelyweg, EA Almelo, The Netherlands) using $\mathrm{Cu} \mathrm{K} \alpha$ radiation $(40 \mathrm{kV}, 45 \mathrm{~mA})$. The experiment temperature was $293 \pm 1 \mathrm{~K}$. Small angular steps of $2 \theta=0.03^{\circ}$ were used to measure the intensity of each Bragg diffraction peak. The grain size and micro-strain were calculated from the full width at half maximum intensity of the measured Bragg diffraction peaks using the ScherrerWilson equation [16]. Phase information on the surface layer of the treated samples was obtained by analyzing corresponding selected-area electron diffraction (SAED) patterns using a TECNAI G20 transmission electron microscope. Thin foil samples for the TEM observations were cut from the treated surface layer by the electro-spark discharge technique. The TEM samples were ground and mechanically polished. The samples were then thinned by ion thinning at low temperature. The micro-hardness values from the surface to the interior were measured on the cross-section of the sample by using a Wolpert L101 MVD Vickers microhardness tester with a load of $50 \mathrm{~g}$ and a duration time of $10 \mathrm{~s}$. The distance between any two neighbouring indentations was at least $10 \mu \mathrm{m}$. The microhardness values were calculated by measuring the geometry of the Vickers pyramid impression. Friction and wear experiments were performed at ambient laboratory conditions $\left(25^{\circ} \mathrm{C}\right.$ and $48 \%$ relative humidity) using a reciprocating sliding wear tester that was equipped with a tangential force sensor on the bottom specimen holder to measure the friction force. The top specimen was a diamond stylus with a $50 \mu \mathrm{m}$ radial spherical tip that slid on the surface of the bottom specimen with a reciprocating distance of $15 \mathrm{~mm}$ and a frequency of 50 cycle $\min ^{-1}$. The test loads were $2,4,6$, and $8 \mathrm{~N}$, 
and the test duration time was $60 \mathrm{~min}$. To compare the friction and wear properties of the surface nano-alloying treated sample with those of the LCS sample, the tests were also performed on specimens cut from the as-annealed sheet under the same conditions.

\section{Results}

Figure 1 shows a cross-sectional morphology via SEM and typical TEM bright-field images at various depths of the surface nano-alloying treated sample. The microstructure and morphology of the astreated surface layer differed from those in the matrix. A homogeneous and continuous surface layer was fabricated on the surface of the LCS after the SNAT. The average thickness of this layer was approximately $35 \mu \mathrm{m}$, which can be clearly distinguished from the LCS substrate. An SPD layer with a thickness of approximately $50 \mu \mathrm{m}$ can be observed beneath the surface layer.

Figure 1b shows a typical TEM image and corresponding selected-area electron diffraction (SAED) patterns of the outermost surface layer of the surface nano-alloying treated sample. This layer was characterized by an ultrafine and equiaxed grain morphology, and the crystallographic orientations were random. The average grain size in this layer was approximately $55 \mathrm{~nm}$. The continuous ring pattern observed in the SAED micrograph is consistent with the observation that the grain size was refined to the nanoscale regime. Based on these observations, the SPD layer can be defined as an nc layer [9]. It can be concluded that the continuous surface layer was 304 stainless steel based on the analysis and calibration of the SAED patterns. Figure 1c shows the TEM bright-field image and corresponding SAED patterns from the substrate at a depth of $50 \mu \mathrm{m}$ from the top surface. Dense dislocation networks, such as dislocation tangles and dislocation walls, can be observed below the surface nc layer, and the average grain size was estimated to be approximately $100-200 \mathrm{~nm}$, which is far greater than that in the outermost surface layer. The corresponding SAED patterns presented a discontinuous circular distribution, implying that the grain size was no longer in the nanometre regime at this depth.

The surface chemical composition of the surface nano-alloying treated samples was detected by EDS to determine the coverage range on the 304 stainless steel. One of the largest differences in the chemical composition between the LCS and 304 stainless steel involved the $\mathrm{Cr}, \mathrm{Ni}$, and $\mathrm{Fe}$ element contents. The $\mathrm{Cr}$, and $\mathrm{Ni}$ element contents of the latter were greater than those in the former, and the Fe element content was approximately $25 \%$ less than that in the former. Based on the difference in the $\mathrm{Cr}, \mathrm{Ni}$, and Fe element contents of the two materials, the coverage of the
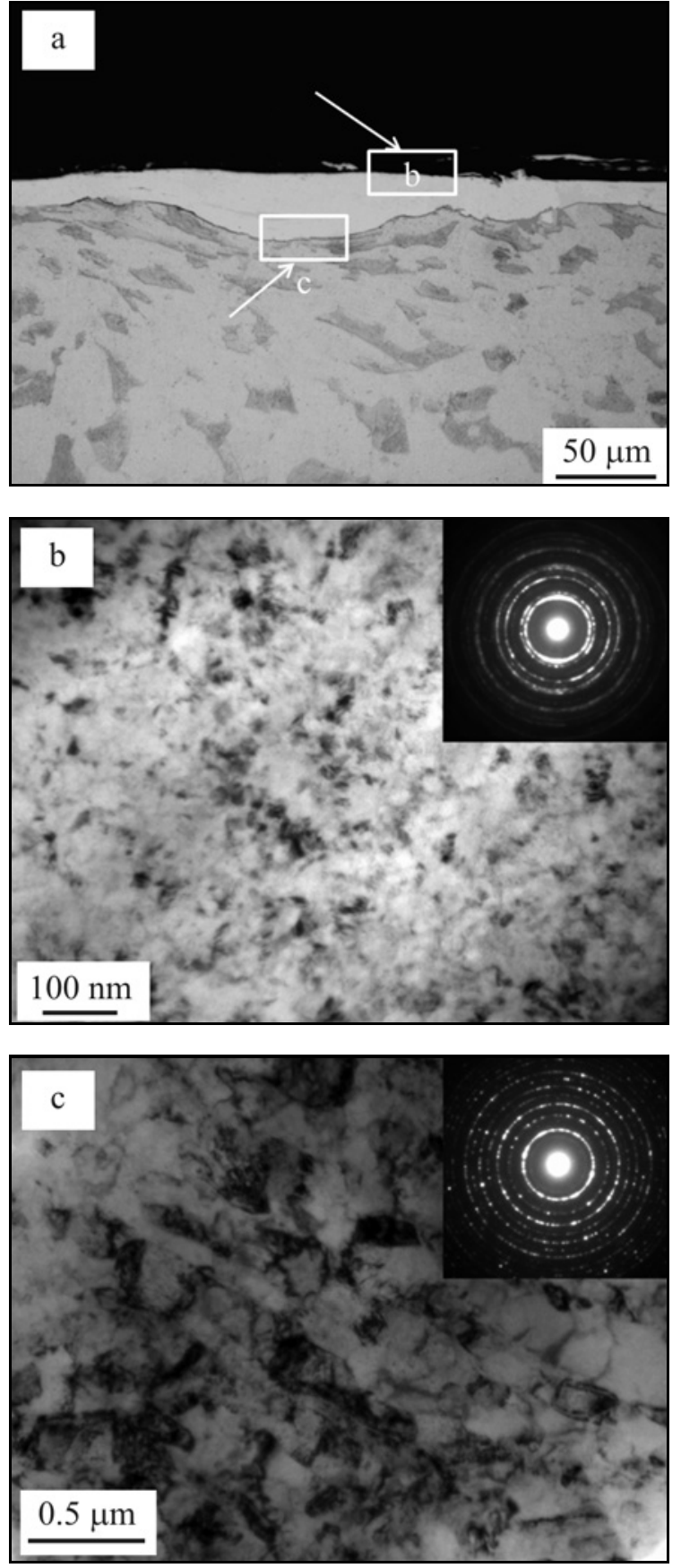

Fig. 1 (a) Cross-sectional morphology via SEM after SNAT for $60 \mathrm{~min}$, (b) the corresponding TEM bright-field image and selected-area electron diffraction (SAED) patterns of the outermost surface layer, and (c) TEM bright-field image and corresponding SAED patterns from the substrate at a depth of $50 \mu \mathrm{m}$ from the top surface.

304 stainless steel can be determined. Figure 2 shows the cross-sectional morphology of the surface nano-alloying treated sample and the corresponding EDS element mapping image. It can be seen that there was a layer comprising $\mathrm{Cr}$ and $\mathrm{Ni}$ in the surface layer after the SNAT. Additionally, the content of Fe in this 

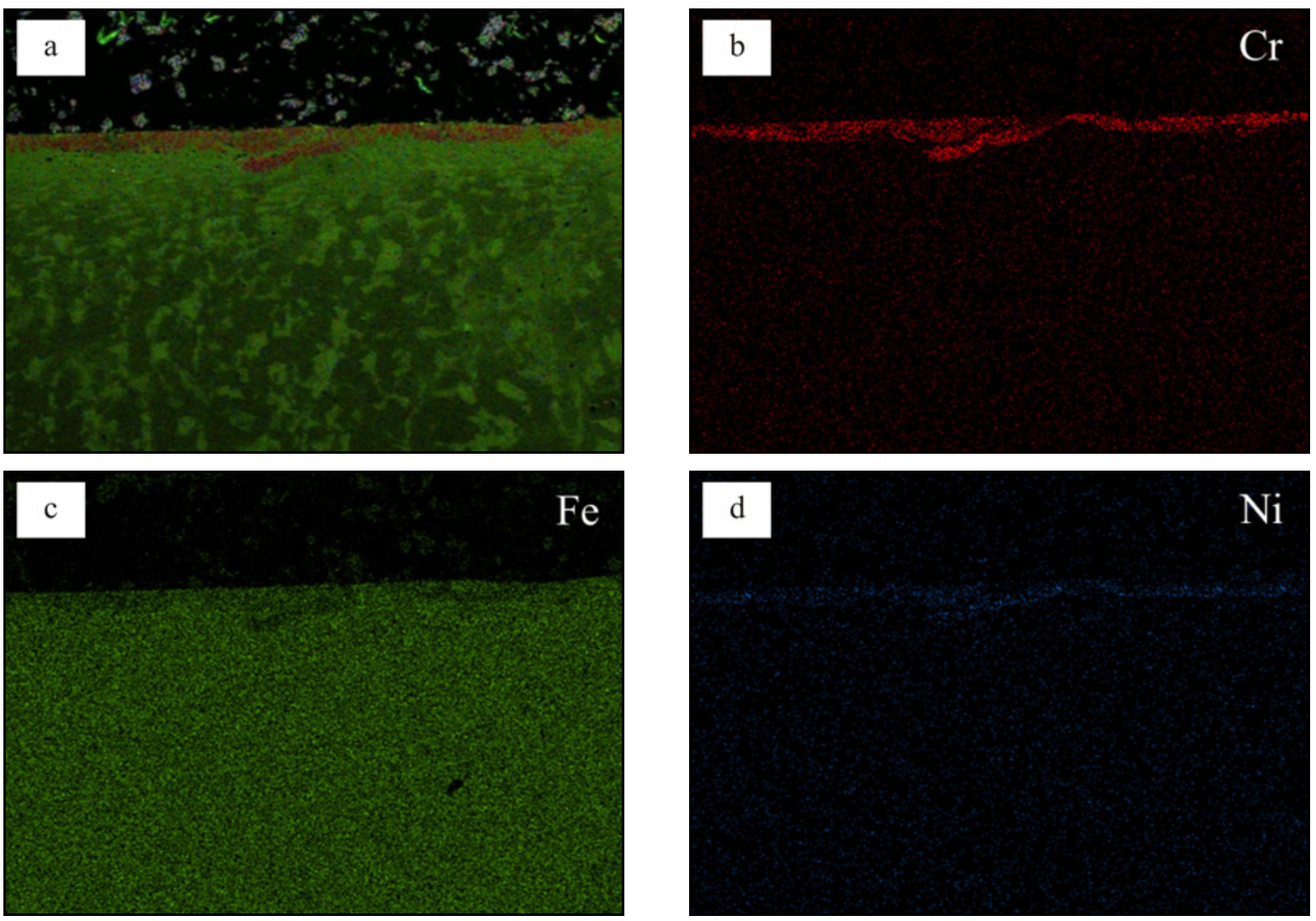

Fig. 2 (a) Cross-sectional morphology of the surface nano-alloying treated sample and the corresponding EDS element mapping images of (b) $\mathrm{Cr}$, (c) Fe, and (d) Ni.

Table 1. Surface chemical composition of the surface nano-alloying treated sample (at.\%)

\begin{tabular}{ccccc}
\hline Element & $\mathrm{Fe}$ & $\mathrm{Cr}$ & $\mathrm{Ni}$ & Percentage \\
\hline 1 & 73.0 & 17.0 & 10.0 & 100 \\
\hline 2 & 74.9 & 15.8 & 9.3 & 93 \\
\hline
\end{tabular}

layer was less than that of the matrix. We can deduce that this layer mainly consisted of 304 stainless steel. The maximum thickness of the layer exceeded $35 \mu \mathrm{m}$ in some regions, and the $\mathrm{Cr}, \mathrm{Ni}$, and $\mathrm{Fe}$ element distributions were uniform.

Figure 3 shows the element mapping image on the surface of the surface nano-alloying treated sample obtained from the corresponding EDS. The $\mathrm{Cr}, \mathrm{Ni}$, and Fe elements were evenly distributed. As shown in Table 1 , the proportion of each element was analyzed based on the EDS results. Analysis No. 1 represents the per cent of $\mathrm{Fe}, \mathrm{Cr}$, and $\mathrm{Ni}$ in the 304 stainless steel, and analysis No. 2 represents the per cent of $\mathrm{Fe}, \mathrm{Cr}$, and $\mathrm{Ni}$ on the surface of the surface nanoalloying treated sample. According to the proportion of $\mathrm{Cr}$ and $\mathrm{Ni}$ in the 304 stainless steel and that of the surface nano-alloying treated sample, the coverage of the 304 stainless steel coating on the surface nano- alloying treated sample surface was calculated to be approximately $93 \%$.

To further investigate the crystallographic phases in the low carbon steel, 304 stainless steel, and surface nano-alloying treated samples, XRD analysis was employed, and the results are shown in Fig. 4. The XRD analysis proved that the surface layers of the three samples were mostly composed of $\alpha$-Fe and $\gamma$-Fe separately. The Bragg diffraction peaks of the LCS sample appeared at $2 \theta$ values of $44.6^{\circ}, 64.9^{\circ}, 82.3^{\circ}$ and the Bragg diffraction peaks of the 304 stainless steel powder appeared at $2 \theta$ values of $43.7^{\circ}, 50.9^{\circ}, 74.8^{\circ}$, and $90.7^{\circ}$ for the $\gamma$-Fe phase. The Bragg diffraction peaks of the surface nano-alloying treated sample appeared at $2 \theta$ values of $43.7^{\circ}, 64.9^{\circ}$, and $82.3^{\circ}$ for the $\alpha$-Fe and $\gamma$-Fe phases, respectively. There was an overlay of the peaks from the 304 stainless steel in the surface nanoalloying treated sample surface. Comparing the Bragg diffraction peaks of the two (111) and (200) crystal planes, the Bragg diffraction peaks of the surface nano-alloying treated sample presented obvious broadening, indicating that the grain size in the outermost surface grains of the surface nano-alloying treated sample was still at the nanoscale. Moreover, the corresponding SAED patterns still had a continuous circular distribution, which means that grains were nc in nature. The broadening of the Bragg diffraction peaks 

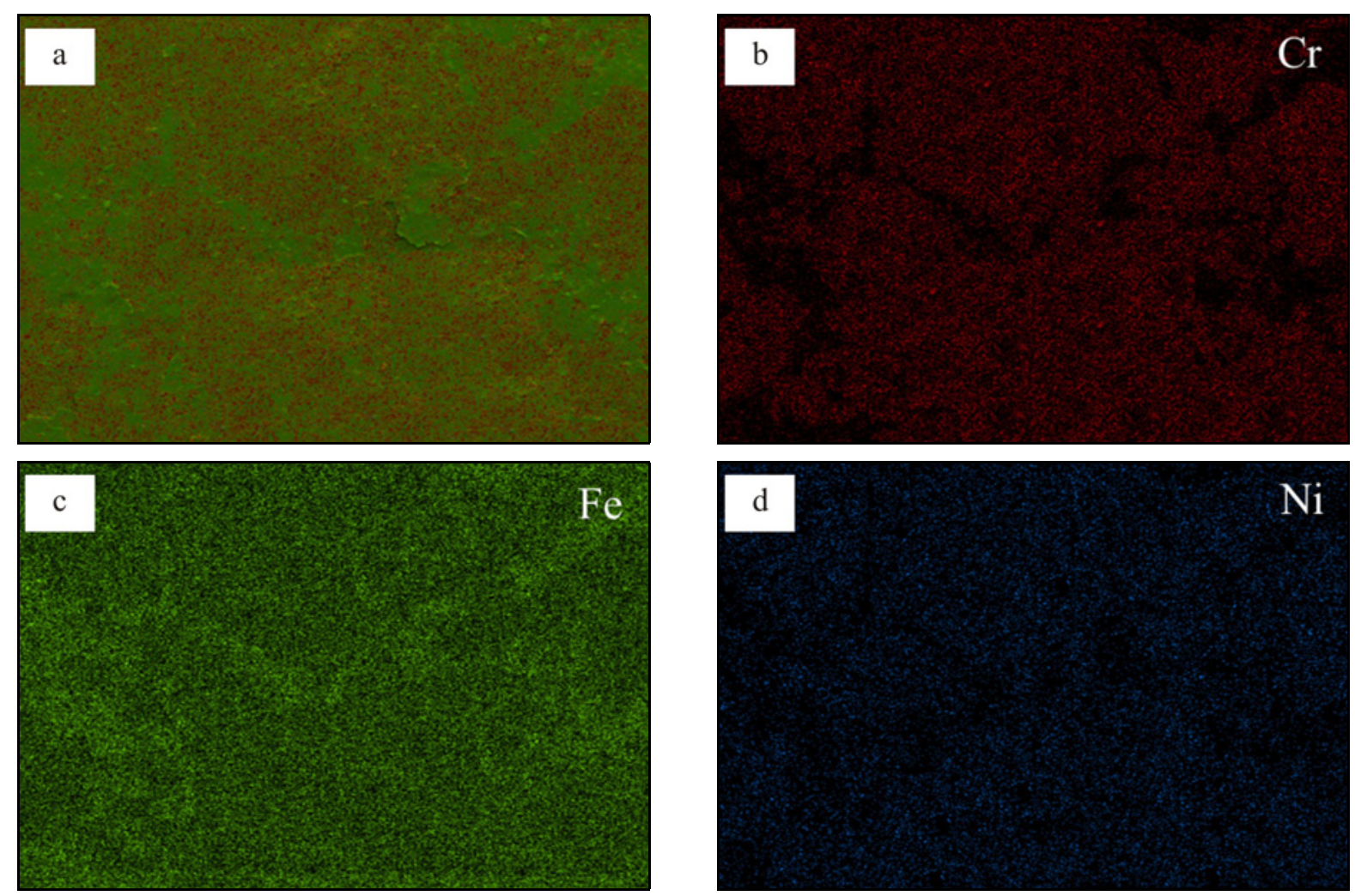

Fig. 3 (a) EDS analysis of the surface nano-alloying treated sample and the corresponding EDS element mapping images of (b) Cr, (c) Fe, and (d) Ni.

in the surface nano-alloying treated sample can be attributed to grain refinement, lattice deformation, and micro-strain development. The approximate function method was used to calculate the grain size and microstrain. According to Scherrer's formula:

$$
\begin{gathered}
\beta_{\mathrm{L}}=\frac{K \lambda_{\mathrm{K} \alpha 1}}{D \cos \theta}, \\
\beta_{\mathrm{G}}=4\left(\varepsilon^{2}\right)^{1 / 2} \tan \theta,
\end{gathered}
$$

where $\theta$ is the Bragg diffraction peak for a given crystal plane, $K$ is a constant related to the grain shape and crystal plane, which was considered to be 0.89 herein, $\beta_{\mathrm{L}}$ is the broadening due to grain refinement, and $\beta_{\mathrm{G}}$ is the broadening due to microstrain. When the grain size is less than $100 \mathrm{~nm}$, the broadening caused by stress can be neglected compared with that caused by the grain size. In this case, the Scherrers' formula was applicable. The grain size was $78.2 \mathrm{~nm}$, and the microstrain was determined to be $0.307 \%$ by using the relative position and full width at half maximum of the XRD peak from the surface nano-alloying treated sample.

The variation in the micro-hardness along depth from the outermost surface layer to the matrix in the cross-section of the surface nano-alloying treated

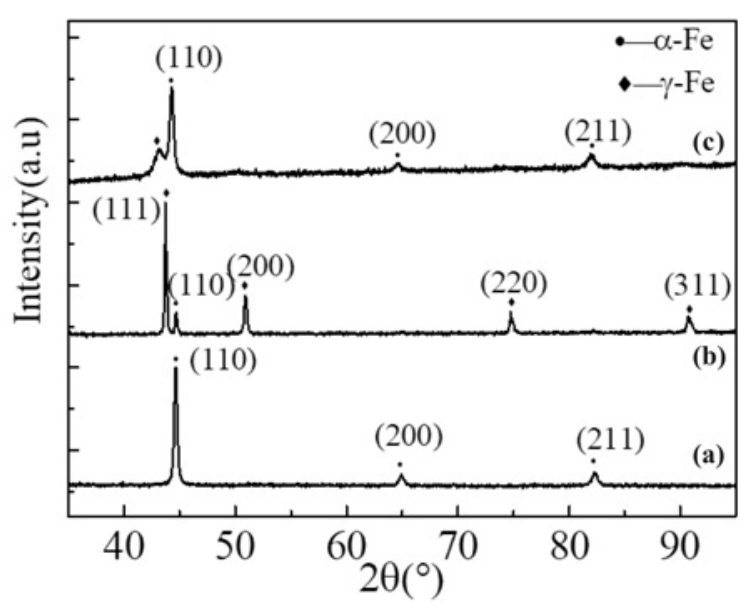

Fig. 4. XRD pattern of the (a) low carbon steel, (b) 304 stainless steel, and (c) surface nano-alloying treated samples.

LCS can be seen in Fig. 5. The average microhardness of the coarse-grained sample was $200 \mathrm{HV}$ as a result of repeated measurements. The microhardness of the surface nano-alloying treated sample was $645 \mathrm{HV}$, which is 3 times higher than that of the matrix. Additionally, it can be seen clearly that the microhardness of the surface nano-alloying treated sample decreased gradually along the depth from the outermost 


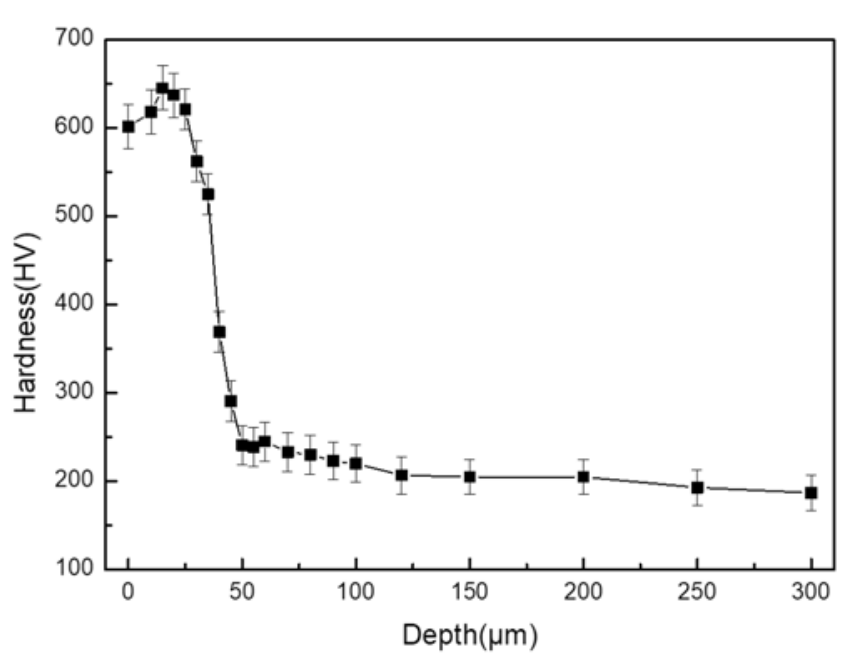

Fig. 5. The variation in the hardness along the depth of the surface nano-alloying treated samples.

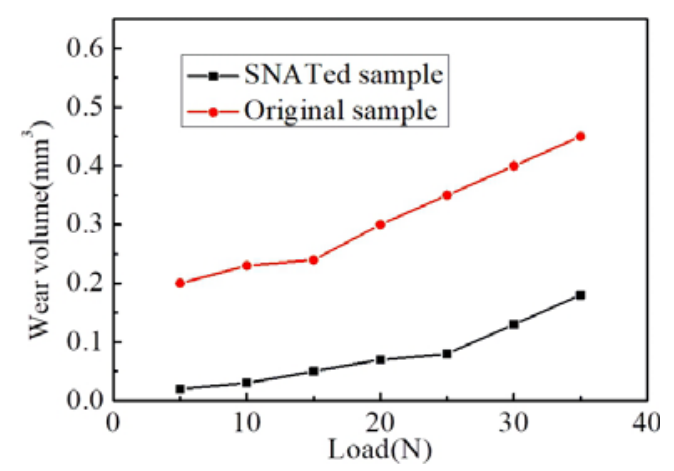

Fig. 6. Variations in the wear volume loss with load for both the surface nano-alloyed treated and the LCS samples.

surface into the matrix. The increased microhardness was mainly attributed to grain refinement in the surface layer following the classical Hall-Petch relationship [13-15]. Furthermore, the hardness sharply decreased at a depth of $50 \mu \mathrm{m}$, and the hardness value became nearly the same as that of the matrix.

Figure 6 shows the variation in the wear volume loss as a function of the load for the surface nanoalloying treated sample and the original LCS sample. For both samples, the wear volume loss increased with increasing load. Still, the wear volume of the surface nano-alloying treated sample was much lower than that of the original LCS sample under each tested load, especially under the load between 25 and $35 \mathrm{~N}$. This indicates that the wear resistance of the LCS was improved by the SNAT. Compared with that of the original LCS sample, the improvement in the wear resistance for the surface nano-alloying treated sample may result from an increase in the hardness in

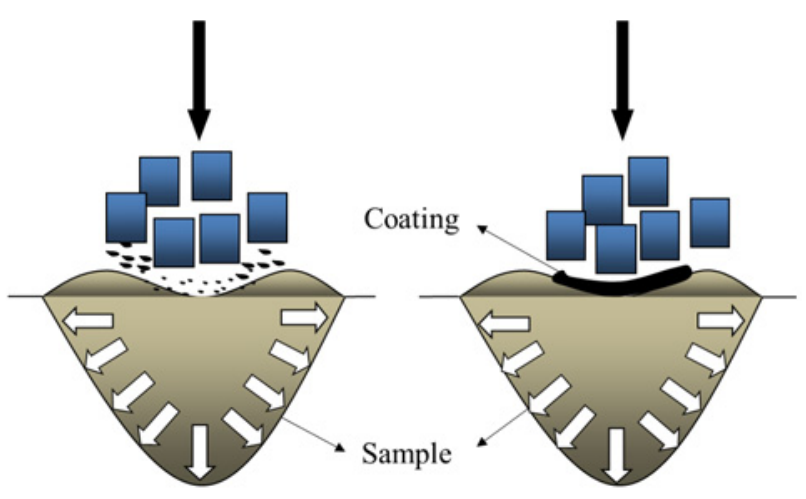

Fig. 7. Schematic illustration of the formation of the stainless steel layer by the SNAT.

the nc surface layer. Simultaneously, the wear volume loss caused by plowing was less than that of the original sample under the same load. As shown in Fig. 6, the dominant wear loss on the surface of the surface nano-alloying treated sample was caused by plowing. When the tested load increased, the dominant wear loss was caused by plastic removal and surface fatigue fracture. Additionally, the increase in the wear volume loss was elevated at large loads than at small loads. The present results clearly showed that an nc surface layer with improved friction and wear properties could be fabricated on an LCS plate by means of a SNAT. This finding is practically important because SNATs are appropriate to strengthen engineering materials and improve the friction and wear properties of the materials.

\section{Discussion}

During the SNAT, the 304 stainless steel powder was bonded to the LCS surface when they were subjected to peening by the steel balls. This SPD resulted in grain refinement. With an increase in the SNAT duration time, the refined 304 stainless steel particles were gradually welded on the surface of the LCS and formed a covering layer with a certain thickness. A schematic illustration of the formation of the stainless steel layer is shown in Fig. 7.

During the SNAT, the projectile collided with the sample surface at a high frequency from different directions and at high speeds. Each collision created plastic deformation near the surface of the sample, and both the strain and strain rate decreased with increasing depth from the top surface. For materials with deformation twins as the main deformation mode, a high strain rate is conducive to the formation of deformation twins, and the large strain increases the twin density. The resulting microstructure presented gradient changes along the thickness direction, and the grain 
size increased with increasing depth. The surface layer can be divided into a nanostructure layer, submicron structure layer and plastic deformation layer.

The hardness of the surface nano-alloying treated sample was mainly attributed to two factors. First, an SPD layer was formed on the material surface. A large number of nc boundaries and dislocation substructures were induced during the SNAT. This significantly increased the structural defects, such as vacancies, gap atoms, and layer dislocations, which hindered the dislocation movement, leading to work hardening and an increase in the hardness. Second, the microstructure refined after the SNAT, and an nc layer was produced. This resulted in an increase in the hardness of the sample. The grain size increased gradually along the depth, and the hardness decreased gradually. This indicated that the main factor for the sample strengthening was the grain refinement, which is consistent with the Hall - Petch formula. The improvement in the friction and wear properties may be attributed to the harder nc surface layer.

The measurement of the hardness variation of the surface nano-alloying treated sample along the depth indicated that the peak in the hardness appeared at approximately $15 \mu \mathrm{m}$ from the top surface rather than at the surface of the sample. It was supposed that the 304 stainless steel powder was continuously buffeted by the steel ball during the SNAT. During this process, micro forging, crushing and cold welding frequently occurred, leading to the formation of blocks and flakes. These blocks and flakes were welded to the sample surface. A small number of cold welding cracks can be observed on the surface of the sample, and the structure approximately $15 \mu \mathrm{m}$ from the surface was very similar; the hardness value reached its peak in this area. There was a specific difference between the statistical average grain size $(55-65 \mathrm{~nm})$ obtained from the TEM bright-field image and the average grain size calculated from the X-ray diffraction peak broadening $(78.2 \mathrm{~nm})$. This result can be attributed to the fact that the XRD patterns presented phase information over the thickness range of $0-20 \mu \mathrm{m}$ from the outermost surface to the interior. At the same time, the TEM bright-field image indicated the phase information for the outermost surface layer (less than $1 \mu \mathrm{m}$ from the surface).

\section{Conclusions}

A surface nc layer comprising 304 stainless steel without impurities was fabricated through the covering of the LCS sample by 304 stainless steel using SMAT. Relevant findings of the study are the following:

1. A continuous smooth covering layer of 304 stainless steel was fabricated on the surface of the LCS us- ing the SNAT. The layer was approximately $35 \mu \mathrm{m}$ in thickness that can be clearly distinguished from the substrate. The coverage of the surface by this layer was $93 \%$. The average grain size at the surface of the sample was $78.2 \mathrm{~nm}$, and the microstrain was $0.307 \%$ based on the relative position and half-height width of the XRD peak obtained on the surface nano-alloying treated sample.

2. Based on the TEM observations, the average grain size in the top layer was in the range of 55$65 \mathrm{~nm}$ with random crystallographic orientation, and the grain size increased with increasing distance from the surface. An SPD layer with a thickness of approximately $50 \mu \mathrm{m}$ can be observed at the bottom of the covering layer. The surface layer can be divided into a nanostructure layer, submicron structure layer, and plastic deformation layer.

3. The microhardness of the surface nano-alloying treated sample was $645 \mathrm{HV}$ at a depth of $15 \mu \mathrm{m}$, which is 3 times higher than that of the matrix. Additionally, it can be seen that the microhardness of the surface nano-alloying treated sample decreased along the depth from the surface layer to the matrix.

4. The wear volume loss was reduced for the surface nano-alloying treated sample compared with that of the original LCS sample. The improvement in the friction and wear properties of the surface nano-alloying treated sample resulted mainly from the harder nc surface layer, which reduced the degree of plowing under a low load and alleviated plastic removal and surface fatigue fracture under a high load.

\section{Acknowledgement}

This research was supported by the National Natural Science Foundation of China (U1810109).

\section{References}

[1] S. Qu, C. X. Huang, Y. L. Gao, G. Yang, S. D. Wu, Q. S. Zang, Z. F. Zhang, Tensile and compressive properties of AISI 304L stainless steel subjected to equal channel angular pressing, Materials Science and Engineering A 475 (2008) 207-216. doi:10.1016/j.msea.2007.04.111

[2] W. P. Tong, N. R. Tao, Z. B. Wang, J. Lu, K. Lu, Nitriding iron at lower temperatures, Science 299 (2003) 686-688.

https://science.sciencemag.org/content/299/5607/686

[3] H. W. Zhang, L. Wang, Z. K. Hei, G. Liu, J. Lu, K. Lu, Low-temperature plasma nitriding of AISI 304 stainless steel with nano-structured surface layer, Zeitschrift für Metallkunde 94 (2003) 1143-1147. doi:10.3139/146.031143

[4] Z. B. Wang, J. Lu, K. Lu, Chromizing behaviors of a low carbon steel processed by means of surface mechanical attrition treatment, Acta Materialia 53 (2005) 2081-2089. doi:10.1016/j.actamat.2005.01.020 
[5] W. P. Tong, Z. Han, L. M. Wang, J. Lu, K. Lu, Lowtemperature nitriding of $38 \mathrm{CrMoAl}$ steel with a nanostructured surface layer induced by surface mechanical attrition treatment, Surface and Coatings Technology 202 (2008) 4957-4963. doi:10.1016/j.surfcoat.2008.04.085

[6] W. P. Tong, J. Sun, L. Zuo, J. C. He, J. Lu, Study on wear and friction resistance of nanocrystalline $\mathrm{Fe}$ nitriding at low temperature, Wear 271 (2011) 653657. doi:10.1016/j.wear.2010.11.024

[7] X. Wu, N. Tao, Y. Hong, B. Xu, J. Lu, K. Lu, Microstructure and evolution of mechanically-induced ultrafine grain in surface layer of Al-alloy subjected to USSP, Acta Materialia 50 (2002) 2075-2084. doi:10.1016/S1359-6454(02)00051-4

[8] T. Roland, D. Retraint, K. Lu, J. Lu, Enhanced mechanical behavior of a nanocrystallized stainless steel and its thermal stability, Materials Science and Engineering A 445-446 (2007) 281-288. doi:10.1016/j.msea.2006.09.041

[9] Y. M. Wang, K. Wang, D. Pan, K. Lu, K. J. Hemker, E. Ma, Microsample tensile testing of nanocrystalline copper, Scripta Materialia 48 (2003) 15811586. doi:10.1016/S1359-6462(03)00159-3

[10] A. Amanov, I. S. Cho, D. E. Kim, Y. S. Pyun, Fretting wear and friction reduction of $\mathrm{CP}$ titanium and Ti-6Al-4V alloy by ultrasonic nanocrystalline surface modification, Surface and Coatings Technology 207 (2012) 135-142. doi:10.1016/j.surfcoat.2012.06.046

[11] H. W. Chang, P. M. Kelly, Y. N. Shi, M. X. Zhang, Thermal stability of nanocrystallized surface produced by surface mechanical attrition treatment in aluminum alloys, Surface and Coatings Technology 206 (2012) 3970-3980. doi:10.1016/j.surfcoat.2012.03.069

[12] N. R. Tao, Z. B. Wang, W. P. Tong, M. L. Sui, J. Lu, $\mathrm{K}$. Lu, An investigation of surface nanocrystallization mechanism in $\mathrm{Fe}$ induced by surface mechanical attrition treatment, Acta Materialia 50 (2002) 4603-4616. doi:10.1016/S1359-6454(02)00310-5
[13] X Si, B. N. Lu, Z. B. Wang, Aluminizing low carbon steel at lower temperatures, Journal of Material Science \& Technology 25 (2009) 433-436. http://www.jmst.org/EN/Y2009/V25/I04/433

[14] Z. B. Wang, N. R. Tao, W. P. Tong, J. Lu, K. $\mathrm{Lu}$, Diffusion of chromium in nanocrystalline iron produced by means of surface mechanical attrition treatment, Acta Materialia 51 (2003) 4319-4329. doi:10.1016/S1359-6454(03)00260-X

[15] W. P. Tong, C. Z. Liu, W. Wang, N. R. Tao, Z. B. Wang, L. Zuo, J. C. He, Gaseous nitriding of iron with a nano-structured surface layer, Scripta Materialia 57 (2007) 533-536. doi:10.1016/j.scriptamat.2007.05.017

[16] Y. L. An, H. Y. Du, Y. H. Wei, N. Wang, L. F. Hou, W. M. Lin, Interfacial structure and mechanical properties of surface iron-nickel alloying layer in pure iron fabricated by surface mechanical attrition alloy treatment, Materials \& Design 46 (2013) 627633. doi:10.1016/j.matdes.2012.11.005

[17] J. Sun, Q. T. Yao, Fabrication of microalloy nitrided layer on low carbon steel by nitriding combined with surface nano-alloying pretreatment, Coatings 6 (2016) 63. doi:10.3390/coatings6040063

[18] S. Romankov, W. Sha, S. D. Kaloshkin, K. Kaevitser, Fabrication of Ti-Al coatings by mechanical alloying method, Surface and Coatings Technology 201 (2006) 3235-3245. doi:10.1016/j.surfcoat.2006.06.044

[19] J. Sun, Q. T. Yao, W. P. Tong, X. D. Du, Y. C. Wu, Simultaneous nitriding for two components of $\mathrm{Ti} /$ steel clad sheet, Surface Engineering 31 (2015) 605-611. doi:10.1179/1743294415Y.0000000072 